\title{
Stochastic modelling and simulations for solute transport in porous media
}

\author{
Tianhai Tian* Kevin Burrage ${ }^{\dagger} \quad$ Ray Volker ${ }^{\ddagger}$
}

(Received 8 August 2003; revised 30 January 2004)

\begin{abstract}
A stochastic model for solute transport in aquifers is studied based on the concepts of stochastic velocity and stochastic diffusivity. By applying finite difference techniques to the spatial variables of the stochastic governing equation, a system of stiff stochastic ordinary differential equations is obtained. Both the semi-implicit Euler method and the balanced implicit method are used for solving this stochastic system. Based on the Karhunen-Loeve expansion, stochastic processes in time and space are calculated by means of a spatial correlation matrix. Four types of spatial correlation matrices are presented based on
\end{abstract}

*Advanced Computational Modelling Centre, The University of Queensland, Brisbane, Queensland 4072, Australia. mailto: tian@maths.uq.edu.au

${ }^{\dagger}$ Department of Mathematics, The University of Queensland, Brisbane, Queensland 4072, Australia. mailto:kb@maths.uq.edu.au

${ }^{\ddagger}$ Department of Civil Engineering, The University of Queesnland, Brisbane, Queensland 4072, Australia. mailto:r.volker@mailbox.uq.edu.au

See http://anziamj.austms.org.au/V45/CTAC2003/Tian for this article, (c) Austral. Mathematical Soc. 2004. Published June 29, 2004. ISSN 1446-8735 
the hydraulic properties of physical parameters. Simulations with two types of correlation matrices are presented.

\section{Contents}

1 Introduction

C552

2 Stochastic model

C554

3 Stochastic differential equations

C556

4 Numerical results

C560

5 Conclusions

C563

References

C563

\section{Introduction}

In the last few decades solute transport in porous media has attracted much attention. This research topic is very important in both environmental protection and resource management. However, due to the inaccuracy or insufficiency of hydrogeological and hydrochemical information, there are many uncertainties in modeling solute transport by the deterministic advectiondispersion equation. To overcome these difficulties, researchers usually use stochastic approaches that treat aquifer properties and flow variables as spatial random fields characterised by a limited number of statistical parameters rather than by well defined deterministic values. Dagan [3] considered the concentration of a solute as a random variable and presented a stochastic model with random coefficients. Deterministic equations are derived for the 
moments of the concentration. The weakness of this type of stochastic approach is the limitation on the magnitude of the random parameters required by the perturbation expansion solution. Rubin [8] recently surveyed the development in this research direction.

Another approach is to simulate the stochastic governing equation directly if the number of stochastic sources is more than one. Hydraulic properties may be based on stochastic coefficients, a stochastic driving force, and/or stochastic boundary and initial conditions [5, 9]. The source of randomness is used more directly in order to deal with larger random variations. This numerical approach not only provides statistical properties of the concentration but also gives stochastic simulations of the solute transport. With the progress in computing facilities and numerical methods for stochastic differential equations, this approach is becoming more and more attractive.

In the stochastic simulation approach, stochastic ordinary differential equations (SODEs) are obtained after applying a finite difference scheme to spatial variables of the stochastic governing equation. One of the difficulties in designing effective numerical methods for strong solutions of SODEs is the stability requirements when the SODEs are stiff in both the deterministic and stochastic components. There have been some attempts in the literature for improving stability properties of numerical methods for stiff SODEs [2]. However, due to the complexity of the SODEs in this paper, the semi-implicit Euler method and the balanced implicit method will be used in simulations.

The outline of this paper is as follows. In Section 2 we first discuss the stochastic governing equation. In Section 3 we apply a semi-discretization process to the stochastic governing equation and obtain a system of soDEs. The calculation of stochastic processes in time and space is also discussed in this section. Numerical simulations are presented in Section 4. 


\section{Stochastic model}

For a three-dimensional deterministic problem, the solute transport in an aquifer is described by the advection-dispersion equation [1]

$$
\frac{\partial(\varphi C)}{\partial t}+\operatorname{div}(\varphi \mathbf{J})=0,
$$

with $\mathbf{v}=-(K / \varphi) \nabla \phi$ and $\mathbf{J}=\mathbf{v} C-\mathbf{D} \nabla C$. Here $C$ is the solute concentration at position $\left(x_{1}, x_{2}, x_{3}\right)$ and time $t, \mathbf{D}$ is a dispersion tensor which accounts for the molecular diffusion and pore-scale dispersion, $\mathbf{v}=\left(v_{x_{1}}, v_{x_{2}}, v_{x_{3}}\right)^{\top}$ is the Darcy velocity vector, $\nabla \phi$ is the gradient of piezometric head, $\varphi$ is the porosity (volumetric water content) and $K$ is the hydraulic conductivity.

In an isotropic porous medium, if aligning an axis with the direction of the velocity vector, the diffusivity matrix can be written as a diagonal matrix $\mathbf{D}=\operatorname{diag}\left(D_{x_{1}}, D_{x_{2}}, D_{x_{3}}\right)$. For the 3 -dimensional steady flow in homogeneous and isotropic soil, it is assumed that $K$ and $\varphi$ are constant, $\mathbf{D}$ is a constant tensor, and $\nabla \phi$ satisfies

$$
\frac{\partial^{2} \phi}{\partial x_{1}^{2}}+\frac{\partial^{2} \phi}{\partial x_{2}^{2}}+\frac{\partial^{2} \phi}{\partial x_{3}^{2}}=0 .
$$

Substituting $\mathbf{v}$ and $\mathbf{J}$ into the governing equation (1) and applying the homogeneous and isotropic condition (2), the following equation gives the 3dimensional solute transport in homogeneous and isotropic aquifers

$$
\frac{\partial C}{\partial t}=-\sum_{i=1}^{3} v_{x_{i}} \frac{\partial C}{\partial x_{i}}+\sum_{i=1}^{3} D_{x_{i}} \frac{\partial^{2} C}{\partial x_{i}^{2}} .
$$

Since the velocity of a solute particle is central in formulating a model of transport process, we first consider the concept of stochastic velocity for the motion of a tracer in a porous medium with uncertainties due to the pore structure:

$$
\overline{\mathbf{v}}=-\frac{K}{\varphi} \nabla \phi+\mathbf{s}_{1},
$$


where $\mathbf{s}_{1}(x, t)=\left(s_{11}, s_{12}, s_{13}\right)^{\top}$ is a stochastic vector in time and space.

Apart from the velocity of a tracer, another important physical phenomenon involved in the solute transport is dispersion which is used to describe solute movement carried by the convection fluid. Dispersion may play a significant role in describing the solute transport if the velocity is very small. The second stochastic concept considered here is the stochastic dispersion

$$
\overline{\mathbf{D}}=\mathbf{D}+\mathbf{s}_{2},
$$

where the stochastic components $\mathbf{s}_{2}(t, x)=\operatorname{diag}\left(s_{21}, s_{22}, s_{23}\right)$ has the same structure of D. Substituting (4) and (5) into (3), the following random field gives the solute transport in porous media with uncertainties:

$$
\frac{\partial C}{\partial t}=-\sum_{i=1}^{3}\left(v_{x_{i}}+s_{1 i}\right) \frac{\partial C}{\partial x_{i}}+\sum_{i=1}^{3}\left(D_{x_{i}}+s_{2 i}\right) \frac{\partial^{2} C}{\partial x_{i}^{2}} .
$$

Due to the limit of space, here we just consider simulations for onedimensional solute transport in an homogeneous and isotropic aquifer. For this one-dimensional problem, the stochastic governing equation is

$$
\frac{\partial C}{\partial t}=-\left(v+s_{1}\right) \frac{\partial C}{\partial x}+\left(D+s_{2}\right) \frac{\partial^{2} C}{\partial x^{2}} .
$$

Note that model (7) is different from that proposed by Kulasiri [5] in which the derivatives of stochastic processes were considered.

The initial condition for 1-dimensional solute transport is $C(x, 0)=c_{0}(x)$ $(0 \leq x \leq l)$ and we consider the upstream boundary condition

$$
C(0, t)=\left\{\begin{array}{ccc}
c_{1}(t), & 0<t<t_{0},\left.\quad \frac{\partial C}{\partial x}\right|_{x=l}=c_{2}(t) . \\
0, & t>t_{0},
\end{array}\right.
$$




\section{Stochastic differential equations}

For a given equidistant mesh on $[0, l]$ with $x_{n}=n h_{x}(n=0,1, \ldots, N+1)$, forward and central differences are used to approximate the first and second partial differentials in space, respectively. Denoting $C_{n}=C\left(x_{n}, t\right)$ and $s_{j n}=$ $s_{j}\left(x_{n}, t\right)(j=1,2)$, stochastic equation (7) is approximated by

$$
\frac{d C_{n}}{d t}=-\left(v+s_{1 n}\right) \frac{C_{n+1}-C_{n}}{h_{x}}+\left(D+s_{2 n}\right) \frac{C_{n+1}-2 C_{n}+C_{n-1}}{h_{x}^{2}},
$$

for $n=1, \ldots, N$. Denoting $\overline{\mathbf{C}}=\left(C_{1}, \ldots, C_{N}\right)^{\top}$ and $\overline{\mathbf{s}}_{j}=\left(s_{j 1}, \ldots, s_{j N}\right)^{\top}$, equations (9) become

$$
d \overline{\mathbf{C}}=\left(\mathbf{A}_{0}+A_{1} \overline{\mathbf{C}}\right) d t+\sum_{n=1}^{N}\left[B_{1 n} d \xi_{1 n}+B_{2 n} d \xi_{2 n}\right] \overline{\mathbf{C}}+\mathbf{A}_{2},
$$

where

$$
\begin{aligned}
\mathbf{A}_{0} & =\left(\frac{D}{h_{x}^{2}} C_{0}, 0, \ldots, 0, \frac{D-v h}{h_{x}^{2}} C_{N+1}\right)^{\top} \\
A_{1} & =\frac{1}{h_{x}^{2}} \operatorname{tri}-\operatorname{diag}\left(D, a_{1}, a_{2}\right) \\
\mathbf{A}_{2} & =\frac{1}{h_{x}^{2}}\left(C_{0} d \xi_{21}, 0, \ldots, 0\right)^{\top}
\end{aligned}
$$

with $a_{1}=v h-2 D$ and $a_{2}=D-v h$. Here $d \xi_{j n}=s_{j n} d t$ and $\xi_{j n}=\xi_{j}\left(x_{n}, t\right)$. $C_{0}$ and $C_{N+1}$ are determined by the boundary conditions. The sparse matri- 
ces $B_{j n}=\left(b_{k l}^{(j n)}\right)_{N \times N}$ have non-zero elements:

$$
\begin{aligned}
& b_{k l}^{(1 n)}=\frac{1}{h_{x}}\left\{\begin{aligned}
-1, & k=n, l=n+1, \quad n=1, \ldots, N-1 ; \\
1, & k=l=n,
\end{aligned}\right. \\
& b_{k l}^{(21)}=\frac{1}{h_{x}^{2}}\left\{\begin{array}{cl}
-2, & k=l=1, \\
1, & k=1, l=2 ;
\end{array}\right. \\
& b_{k l}^{(2 n)}=\frac{1}{h_{x}^{2}}\left\{\begin{array}{cl}
-2, & k=l=n, \\
1, & k=n, l=n-1 \text { or } l=n+1, \quad n=2, \ldots, N-1 ;
\end{array}\right. \\
& b_{k l}^{(2 N)}=\frac{1}{h_{x}^{2}}\left\{\begin{aligned}
1, & k=N, l=N-1, \\
-1, & k=N, l=N .
\end{aligned}\right.
\end{aligned}
$$

Now we consider two different simulation methods for solving soDEs. For the general SODE in Itô form

$$
d y=g_{0}(y) d t+\sum_{j=1}^{d} g_{j}(y) d W_{j}(t)
$$

the semi-implicit Euler method takes the form [2]

$$
y_{i+1}=y_{i}+g_{0}\left(y_{i+1}\right) h_{t}+\sum_{j=1}^{d} \Delta W_{j}^{(i)} g_{j}\left(y_{i}\right) .
$$

Here the $\Delta W_{j}^{(i)}$ are the Wiener increments for time step $i+1$ and are independent $N\left(0, h_{t}\right)$. The balanced implicit method takes the form [7]

$$
y_{i+1}=y_{i}+g_{0}\left(y_{i}\right) h_{t}+\sum_{j=1}^{d} \Delta W_{j}^{(i)} g_{j}\left(y_{i}\right)+U_{i}\left(y_{i}-y_{i+1}\right),
$$

with

$$
U_{i}=u_{0}\left(y_{i}\right) h_{t}+\sum_{j=1}^{d}\left|\Delta W_{j}^{(i)}\right| u_{j}\left(y_{i}\right)
$$


Here $u_{j}(j=0,1, \ldots, d)$ represent $m \times m$-matrix-value functions if $y$ is a $m$-dimensional vector. These matrices are chosen appropriately to get good damping properties. Applying the semi-implicit Euler method (12) to (10) gives

$$
\overline{\mathbf{C}}_{i+1}=\overline{\mathbf{C}}_{i}+\left(\mathbf{A}_{0}+A_{1} \overline{\mathbf{C}}_{i+1}\right) h_{t}+\sum_{n=1}^{N}\left[B_{1 n} \Delta \xi_{1 n}^{(i)}+B_{2 n} \Delta \xi_{2 n}^{(i)}\right] \overline{\mathbf{C}}_{i}+\Delta \mathbf{A}_{2},
$$

where $\overline{\mathbf{C}}_{i}=\overline{\mathbf{C}}\left(t_{i}\right)$ with $t_{i}=i h_{t}$, and $\Delta \mathbf{A}_{2}=\left(C_{0} \Delta \xi_{21} / h_{x}^{2}, 0, \ldots, 0\right)^{\top}$.

Using the Karhunen-Loeve expansion [4], the stochastic process $\Delta \xi(x, t)=$ $\xi\left(x, t+h_{t}\right)-\xi(x, t)$ has the truncating expansion

$$
\Delta \xi(x, t)=\sum_{n=1}^{N} \sqrt{\lambda_{n}} \Delta \eta_{n}(t) f_{n}(x)
$$

where the $\Delta \eta_{n}(t) \sim N\left(0, \sqrt{h_{t}}\right)$ are independent Gaussian processes. Then the stochastic vector $\Delta \bar{\xi}(t)=\left(\Delta \xi\left(x_{1}, t\right), \ldots, \Delta \xi\left(x_{N}, t\right)\right)^{\top}$ can be expressed as $\Delta \bar{\xi}(t)=F \Delta \bar{\eta}$ with $F=\left(F_{i j}\right)_{N \times N}=\left(f_{j}\left(x_{i}\right)\right)_{N \times N}$ and

$$
\Delta \bar{\eta}=\left(\sqrt{\lambda_{1}} \Delta \eta_{1}(t), \ldots, \sqrt{\lambda_{N}} \Delta \eta_{N}(t)\right)^{\top} .
$$

A spatial correlation matrix $Q=\left(Q_{i j}\right)_{N \times N}$ is defined with eigenvalues $\lambda_{i}$ and the corresponding eigenvectors $\mathbf{e}_{n}=\left(f_{n}\left(x_{1}\right), \ldots, f_{n}\left(x_{N}\right)\right)^{\top}$. There are four types of spatial correlation matrices based on the hydraulic properties of physical parameters.

Type 1. The spatial independent matrix

$$
Q_{i j}=q \delta_{i j}
$$

Type 2. The adjacent correlation matrix

$$
Q_{i j}=\operatorname{tri-\operatorname {diag}}(q, 2 q, q) .
$$


Type 3. The exponential correlation matrix [4]

$$
Q_{i j}=q \exp \left(-\frac{\left|x_{i}-x_{j}\right|}{r}\right),
$$

with a parameter $r$ for adjusting the correlation decay rate between two points of the process.

Type 4. The triangular correlation matrix [4]

$$
Q_{i j}=q\left(1-r\left|x_{i}-x_{j}\right|\right), \quad\left|x_{i}-x_{j}\right| \in\left[0, \frac{1}{r}\right],
$$

where $r$ is a parameter for adjusting the correlation between two points of the process.

Here $q$ in all four types of correlation matrices is a scalar with different values for the stochastic components of the velocity and diffusivity, respectively.

Applying the balanced implicit method (13) to (10), gives

$$
\begin{aligned}
\overline{\mathbf{C}}_{i+1}= & \overline{\mathbf{C}}_{i}+\left(\mathbf{A}_{0}+A_{1} \overline{\mathbf{C}}_{i}\right) h_{t}+\sum_{n=1}^{N}\left[B_{1 n} \Delta \xi_{1 n}^{(i)}+B_{2 n} \Delta \xi_{2 n}^{(i)}\right] \overline{\mathbf{C}}_{i}+\Delta \mathbf{A}_{2} \\
& +\left\{\bar{A}_{1} h_{t}+\sum_{n=1}^{N}\left[\bar{B}_{1 n}\left|\Delta \xi_{1 n}^{(i)}\right|+\bar{B}_{2 n}\left|\Delta \xi_{2 n}^{(i)}\right|\right]\right\}\left(\overline{\mathbf{C}}_{i}-\overline{\mathbf{C}}_{i+1}\right) .
\end{aligned}
$$

The implicit nature of this method is adjusted by choosing the matrix $\bar{A}_{1}$ and the $\bar{B}_{j n}$ appropriately.

Matrix $\bar{A}_{1}$ is determined by the properties of $A_{1}$. As the eigenvalues of $A_{1}$ are all negative, we choose $\bar{A}_{1}=-A_{1}$. In this case the deterministic component is implicit. For stochastic components, the non-zero elements of 
the matrices $\bar{B}_{j n}=\left(\bar{b}_{k l}^{(j n)}\right)_{N \times N}$ can be chosen as: $\bar{b}_{n n}^{(1 n)}=\mu_{1} / h_{x}$ and $\bar{b}_{n n}^{(2 n)}=$ $\mu_{2} / h_{x}^{2}(n=1, \ldots, N)$. We have that

$$
\sum_{n=1}^{N} \bar{B}_{j n}\left|\Delta \xi_{j n}^{(i)}\right|=\lambda_{j} \operatorname{diag}\left(\left|\Delta \xi_{j 1}\right|, \ldots,\left|\Delta \xi_{j N}\right|\right), \quad j=1,2,
$$

with $\lambda_{1}=\mu_{1} / h_{x}$ and $\lambda_{2}=\mu_{2} / h_{x}^{2}$. With positive $\mu_{1}$ and $\mu_{2}$, it can be proved that the matrix

$$
I-A_{1}+\sum_{n=1}^{N} \bar{B}_{1 n}\left|\Delta \xi_{1 n}^{(i)}\right|+\sum_{n=1}^{N} \bar{B}_{2 n}\left|\Delta \xi_{2 n}^{(i)}\right|
$$

has an inverse and every eigenvalue of the inverse matrix is less than 1 . Compared with the semi-implicit Euler method, the balanced implicit method can be implemented with better stability properties.

\section{Numerical results}

In this section we present stochastic simulations of the solute transport in an aquifer by using the semi-implicit Euler method and the balanced implicit method. Due to the limit of space, we just give simulations with Types 1 and 4 correlation matrices. Hydrology parameters are: Darcy velocity: $v=$ $-\frac{K}{\varphi} \nabla \phi=1 \mathrm{~cm} /$ hour; diffusivity: $D=0.03 \mathrm{~cm}^{2} /$ hour [10], and step sizes are $h_{x}=0.05$ and $h_{t}=0.01$. The initial condition is $C(x, 0)=0(0 \leq x \leq 5)$ and the boundary conditions are $c_{1}(t)=1, t_{0}=3$ and $c_{2}(t)=0$ in (8). The scalars $q$ in (14) are $q_{1}=0.002$ and $q_{2}=0.00002$ while those in (15) are $q_{1}=0.005$ and $q_{2}=0.00005$ for $\Delta \xi_{1 n}$ and $\Delta \xi_{2 n}$, respectively.

Figure 1 gives simulations obtained by the semi-implicit Euler method with the spatial independent matrix (left) and the triangular correlation matrix $(r=1 / l=0.2)$ (right) at $t=1,4,7$. Fluctuations of the concentration in the left figure are larger than those in the right figure. For the triangular 

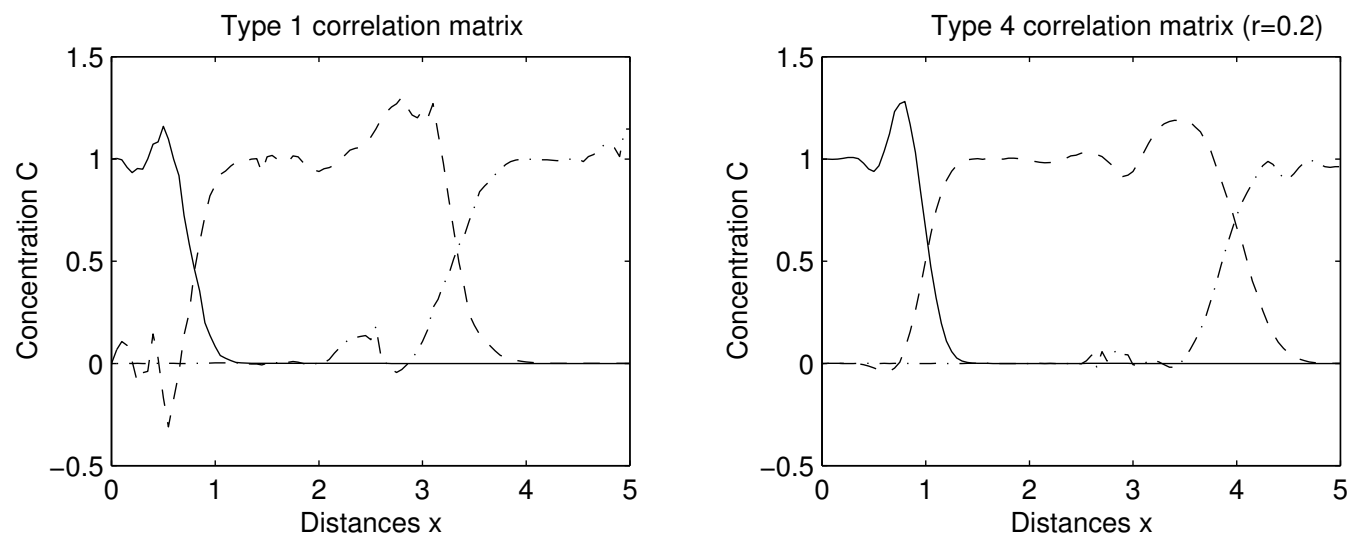

Figure 1: Simulations of the semi-implicit Euler method with two different spatial correlation matrices given by Eqs (14) (left) and (15) (right). $(t=$ 1: solid line; $t=4$ : dashed line; $t=7$ : dash-doted line).

correlation matrix we have also simulated the system with other $r$ in (15). Fluctuations in concentration are larger if a larger $r$ is used.

As concentrations in deterministic simulations are in the range of $[0,1]$, concentrations beyond this range in stochastic simulations may be derived from stochastic processes in the governing equation, the finite difference procedure for discretising the governing equation, and the accuracy and stability properties of numerical methods for solving (10). Here we use the balanced implicit method to study the impact of stability properties of numerical methods on simulation results.

We can choose different $\mu_{1}$ and $\mu_{2}$ in the balanced implicit method to adjust stability properties. Numerical simulations with all four types of spatial correlation matrices indicate that, if larger $\mu_{1}$ and $\mu_{2}$ are used, fluctuations in concentration are smaller but computational errors are larger. If $\mu_{1}$ and $\mu_{2}$ are too small, the difference between simulations obtained by the semi-implicit Euler method and by the balanced implicit method is very 

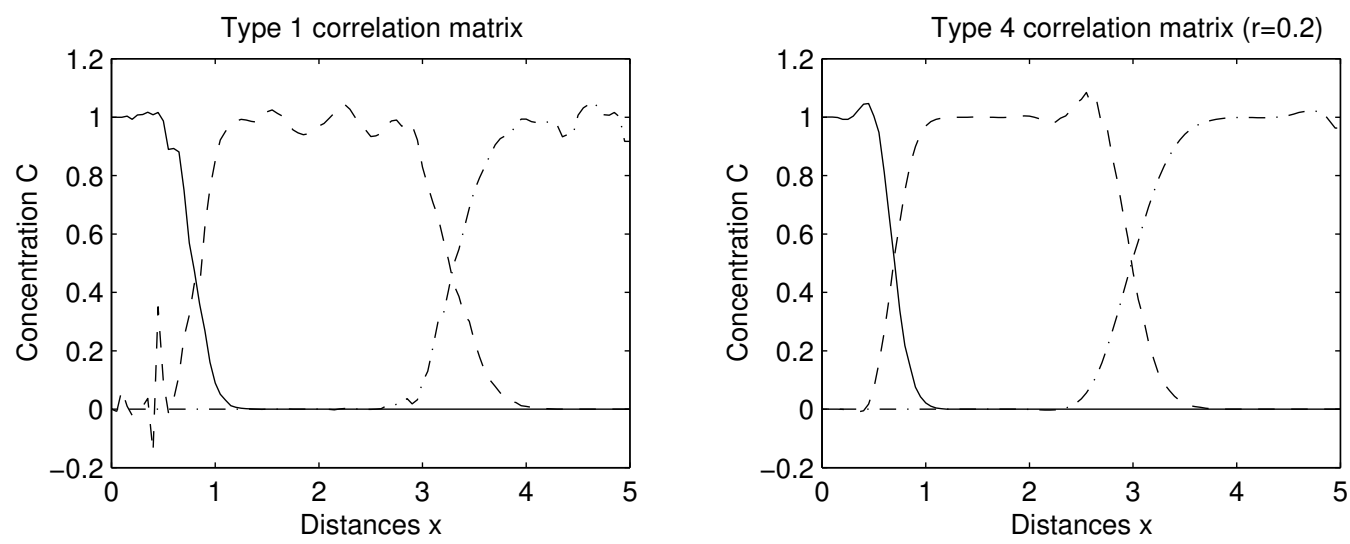

Figure 2: Simulations of the balanced implicit method with two different spatial correlation matrices given by Eqs (14) (left) and (15) (right). $(t=$ 1: solid line; $t=4$ : dashed line; $t=7$ : dash-doted line).

small. For the problem in this paper it is recommended to use $\mu_{1}$ and $\mu_{2}$ in the range between 0.5 and 2 . In this case the stability properties of the balanced implicit method are improved and the computational errors are acceptable.

Figure 2 gives simulations using the balanced implicit method with $\mu_{1}=$ $\mu_{2}=1$. For both types of spatial correlation matrices, negative concentrations in Figure 2 are much smaller than those in Figure 1. Similar observation can also be found for concentration fluctuations which are greater than 1. This indicates that good stability properties of numerical methods have positive impact on fluctuations in stochastic simulations. Thus numerical methods with good stability properties can reduce fluctuations in stochastic simulations. 


\section{Conclusions}

A stochastic model for solute transport in aquifers has been considered based on the concepts of stochastic velocity and stochastic diffusivity. The semiimplicit Euler method and the balanced implicit method have been used to solve the derived soDEs. The drawback of the semi-implicit Euler method is the large non-physical values of concentrations $(C<0$ or $C>1)$. It has been shown that the good stability properties of the balanced implicit method have significant impact on stochastic simulations.

Both the semi-implicit Euler method and the implicit balanced method are of strong order half. Considering the forward difference scheme applied to the governing equation (7), the accuracy of numerical simulations in this paper is just $\mathcal{O}\left(\sqrt{h_{t}}+h_{x}\right)$. In order to improve the accuracy of stochastic simulations, future work should be based on the development of implicit numerical methods for solving soDEs with high convergence order and with better stability properties. In addition it would be very important to employ other discretization schemes for the governing equation (7) in order to improve stability properties, which is a key issue in numerical solutions of partial differential equations. Candidate schemes include the up-wind scheme and the Dufort-Frankel scheme [6]. All of these are the topics for future work.

\section{References}

[1] J. Bear and A. Verrujt, Modeling Groundwater Flow and Pollution: with Computer Programs for Sample Cases, Kluwer Academic Publishers, Dordrecht, 1987. C554

[2] K. Burrage, P. M. Burrage and T. Tian, Numerical Methods for Strong Solutions of Stochastic Differential Equations: an Overview, Proc.

R. Soc. Lon. A 460 (2004) 373-402. C553, C557 
[3] G. Dagan, Flow and transport in porous formations (Springer-Verlag, Berlin, 1989). C552

[4] R. G. Ghanem and P. D. Spanos, Stochastic finite elements: A spectral approach (Springer-Verlag, New York, 1991). C558, C559

[5] D. Kulasiri, Simulation of solute transport in heterogeneous aquifers using stochastic partial differential equations, in: Proceedings of the Congress of Modelling and Simulation, Australia, Volume 2 610-616. C553, C555

[6] D. D. McCracken and W. S. Dorn, Numerical methods and FORTRAN programming, John Wiley and Sons, New York, 1975. C563

[7] G. N. Milstein, E. Platen and H. Schurz, Balanced implicit methods for stiff stochastic systems, SIAM J. Numer. Anal., 35 (1998), 1010-1019. C557

[8] Y. Rubin, Transport of inert solutes by groundwater: recent developments and current issues, in G. Dagan and S. P.Neuman ed., Subsurface Flow and Transport: A Stochastic Approach (Cambridge University Press, 1997) 115-132. C553

[9] T. E. Unny, Stochastic partial differential equations in groundwater hydrology, Part I: Theory, Stochastic Hydrol. Hydraul., 3 (1989), P135-153. C553

[10] R. Zhang, K. Huang and M. T. Genuchten, An efficient Eulerian-Lagrangain method for solving solute transport problem in steady and transient flow fields, Water Resource Research, 28 (1993), 4131-4138. C560 